\title{
Design issues of thermal induced effects and temperature dependent material properties in Abaqus
}

\author{
I. Both ${ }^{1}$, F. Wald ${ }^{1} \&$ R. Zaharia ${ }^{2}$ \\ ${ }^{I}$ Department of Steel and Timber Structures, \\ Czech Technical University in Prague, Czech Republic \\ ${ }^{2}$ Department of Steel Structures and Structural Mechanics \\ Politehnica University of Timisoara, Romania
}

\begin{abstract}
Structural fire design is greatly increasing the use of numerical models for fire resistance assessment. Besides the dedicated software which includes the necessary setup for thermo-mechanical analysis, general purpose software, like Abaqus, is used for uncommon cases which may regard material models or complex behaviour defined by springs. In this paper, the theoretical considerations for material properties are compared to the approaches of computational models. Thermal induced effects in structural analysis by constant temperature and gradient are described starting from basic relations to software input data. The concrete damaged plasticity material model is discussed regarding the behaviour of concrete at elevated temperatures. Simple examples are presented to demonstrate the proper use of material properties and thermal input data.

Keywords: thermal expansion coefficient, temperature gradient, thermomechanical analysis, concrete damaged plasticity.
\end{abstract}

\section{Introduction}

Structures subjected to high temperatures exhibit a wide range of complex phenomena due to the temperature dependent thermal and mechanical properties of materials. Numerical models must consider the change of properties function of temperature. Since temperature development is dependent on time, the 
numerical computation is a transient analysis and must be defined considering an appropriate time step.

Dedicated finite element computer codes for elevated temperature analysis like Safir [1] or Vulcan [2] have a large horizon for fire design simulations and facilitate the input data needed for such analysis. For atypical cases of material models (high strength concrete), flexible support conditions or complex connection behaviour, general purpose software like Abaqus [3] or ANSYS [4] are suitable for numerical simulations.

Fire design codes refer to computer programs as advanced models and they need to be validated against experimental results in order to support the design verifications. Examples can be found in literature for validation of dedicated software [5, 6] as well as for general purpose finite element codes [7]. While the first category includes most of the material properties and settings for the numerical models, the latter require additional information for environment definition.

The present paper intends to present the distinct approaches of Abaqus in a thermo-mechanical analysis needed to support the validation of structures subjected to elevated temperatures. Thermal expansion coefficient, temperature gradient and concrete model must be regarded with special attention due to their distinct definition in Abaqus.

\section{Analytical aspects}

Elements subjected to temperature exhibit a behaviour sensitive to temperature distribution either on longitudinal direction or through cross section.

Thermal expansion coefficient is a quantity that expresses the increase in dimension of a solid due to temperature increase. For a linear increase, the most frequent relation used to obtain the variation of thermal expansion coefficient with temperature is:

$$
\alpha=\frac{d L / L}{d T}
$$

where: $d L / L$ is the thermal elongation and $d T$ is the temperature increase. In structural engineering, a linear element increase usually engages a change in axial force.

For an indeterminate structural analysis, thermal expansion is found in the redundant reaction displacement term as:

$$
D_{t}=\int n_{1} \cdot \alpha \cdot t \cdot d x
$$

where: $D_{t}$ is the displacement at the point of the redundant reaction caused by the temperature increase, $n_{l}$ is the axial force due to unitary redundant force and $t$ is the temperature increase in the element.

Temperature gradient is a quantity that expresses the temperature change direction in degree units per unit length. The physical meaning in structural engineering is commonly regarded as the bending of the element. 
For an indeterminate structural analysis, thermal expansion is found in the redundant reaction displacement term as:

$$
D_{\Delta t}=\int m_{1} \cdot \frac{\alpha \cdot \Delta t}{h} \cdot d x
$$

where: $D_{\Delta t}$ is the displacement at the point of the redundant (moment) reaction caused by the temperature gradient, $m_{l}$ is the bending moment due to unitary redundant force (moment), $h$ is the height of the cross section and $\Delta t$ is the temperature difference between the extreme sides of element cross section.

A concrete model in advanced calculation models has to be defined according to design codes [8]. In thermo-mechanical analysis the structural behaviour is determined by the thermal strain and stress-strain relationship.

Thermal strain of materials for small temperature increase is frequently given as a linear function, therefore, the expansion coefficient which is the derivative with respect to temperature is a constant. For high temperature difference the thermal elongation is not less than a second order function. For concrete, design codes $[8,9]$ give the function presented in Figure 1 according to relations (4).

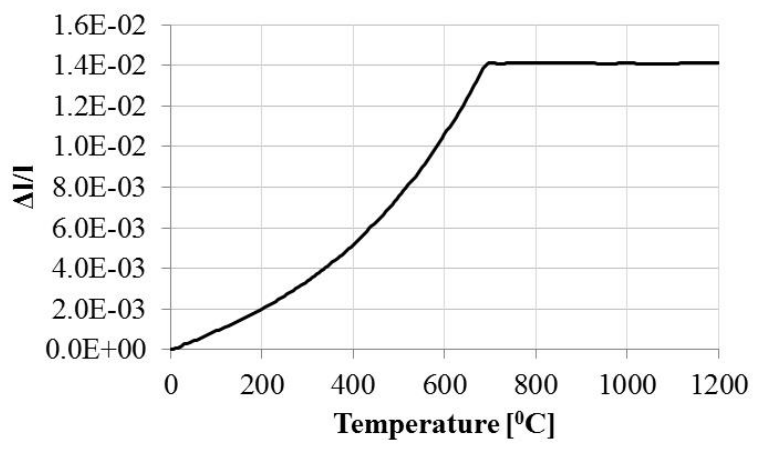

Figure 1: Thermal strain.

$$
\left\{\begin{array}{cc}
\Delta l / l=-1.8 . e^{-4}+9 . e^{-6} \theta+2.3 \cdot e^{-11} \theta^{3} & 20<\theta<700 \\
\Delta l / l=14 . e^{-3} & 700<\theta<1200
\end{array}\right.
$$

where: $\theta$ is the material temperature and the temperature intervals are considered in Celsius degrees.

The constitutive law is based on the following relations for the compressive behaviour:

$$
\sigma_{c \theta}=f_{c \theta}\left[3\left(\frac{\varepsilon_{c \theta}}{\varepsilon_{c u \theta}}\right) /\left\{2+\left(\frac{\varepsilon_{c \theta}}{\varepsilon_{c u}}\right)^{3}\right\}\right]
$$

Mechanical properties at elevated temperature are decreasing according to [9] for both compression and tension. In practice, although often omitted, it is common to set the tensile strength to $10 \%$ of the compressive strength. 


\section{Finite element approach}

It is known that general purpose finite elements codes, do not have a defined unit measurement system and they need to be considered in a consistent manner. In Abaqus, if thermal analysis is involved the absolute zero temperature and Stefan-Boltzmann constant need to be defined $(-273.15$ and $5.67 \mathrm{e}-8$ respectively). By using these values in the numerical model the temperature is considered to be defined in Celsius degrees and for ease of thermal properties use given in codes [8-10] the following units of measurement may be considered: $\mathrm{kg}, \mathrm{m}, \mathrm{s}, \mathrm{N},{ }^{\circ} \mathrm{C}$.

\subsection{Expansion coefficient}

Computing the derivative of thermal strain for concrete using relation (4) will lead to a shape depicted in Figure 2 as incrementation pattern. This pattern may be found in many reports and articles from literature. Abaqus allows the use of temperature dependent expansion coefficient. By creating a simple cantilever model using a beam element of $1 \mathrm{~m}$ subjected to a temperature increase from $20^{\circ} \mathrm{C}$ to $1200^{\circ} \mathrm{C}$ for a step of 1200 s the displacement of the free end in longitudinal direction will follow the shape presented in Figure 3 as the incrementation curve. It may be observed that for the unitary length of cantilever the displacement is far from the expected.

In comparison with this approach, Abaqus computes the thermal elongation with respect to a reference temperature considered the initial temperature of material [Abaqus] which is $0^{\circ} \mathrm{C}$ if not defined otherwise. For structural analysis in civil engineering the initial temperature is frequently considered to be $20^{\circ} \mathrm{C}$ which, in Abaqus, may be set using an initial condition through a predefined field.

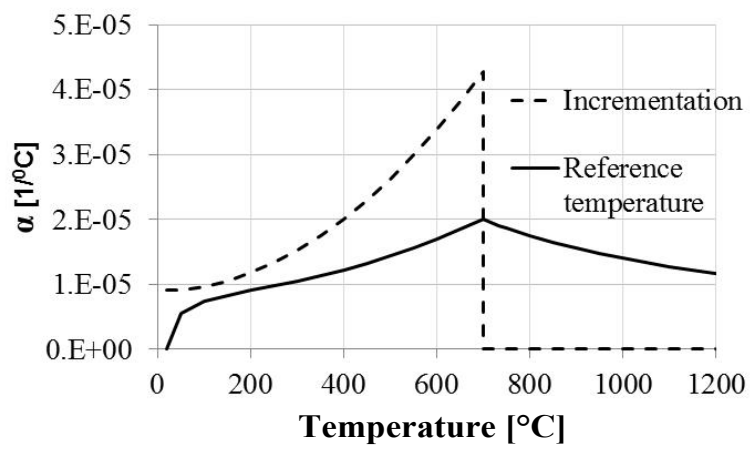

Figure 2: Thermal expansion coefficient.

The relation leading to a thermal expansion coefficient which considers the temperature increase with respect to an initial temperature of $20^{\circ} \mathrm{C}$ is given by:

$$
\alpha=\frac{\Delta l / l}{T}
$$

where: $T$ is the temperature of material. 
Using relation (6) the thermal expansion coefficient variation is depicted in Figure 2 under the reference temperature pattern. By performing the same analysis on a $1 \mathrm{~m}$ cantilever, using a predefined field for an initial temperature of $20^{\circ} \mathrm{C}$ and the values for $\alpha$ resulted from relation (6) the longitudinal displacement obtained are according to the curve presented in Figure 3 as reference temperature.

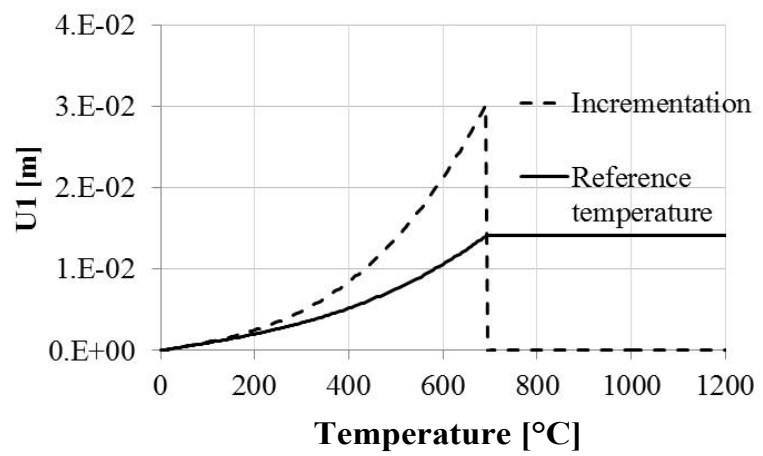

Figure 3: Free end displacement due to temperature increase.

It may be observed that the thermal elongation of a unitary length bar due to temperature increase using the initial temperature as reference for expansion coefficient is according to the relations given in the fire design codes.

\subsection{Temperature gradient}

A discussion on the effects on structural elements may be opened due to temperature difference on cross-section. In Abaqus, beam elements are defined with respect to a reference line. In case of composite structures involving linear elements for beams and surface elements for slabs, it may be required to position the reference line outside the cross section. This may lead to uncertainties in gradient definition through the cross section.

For a time/temperature dependent analysis the input data of a thermal gradient through the cross section in Abaqus is not the simple increase of temperature over a unit of distance. The necessary input data can be found in equation (7).

$$
T_{\text {ref }}\left(1+d_{2} \cdot x\right)=T_{2}
$$

where $T_{\text {ref }}$ is the temperature at reference line level, $d_{2}$ is the distance from reference line to a point along 2-direction, $x$ is the gradient and $T_{2}$ is the temperature at the level of $d_{2}$.

Considering a beam with the same temperature profile, $400^{\circ} \mathrm{C}$ at the bottom and $600^{\circ} \mathrm{C}$ at the top, different gradient values must be defined. First, the reference line temperature is calculated by linear interpolation with respect to geometry of the beam as presented in Figure 4. The two cases, reference line coincide with centroid and reference line having different position from centroid will lead to different values, $500^{\circ} \mathrm{C}$ and $700^{\circ} \mathrm{C}$, respectively. In the numerical 
model these values need to be defined in tabulated data amplitude and used in a predefined field form.

The temperature gradient necessary for the input of numerical model is the value of $x$ obtained by solving equation (7). The two values obtained for the discussed cases are 2 and 1.42857 respectively.
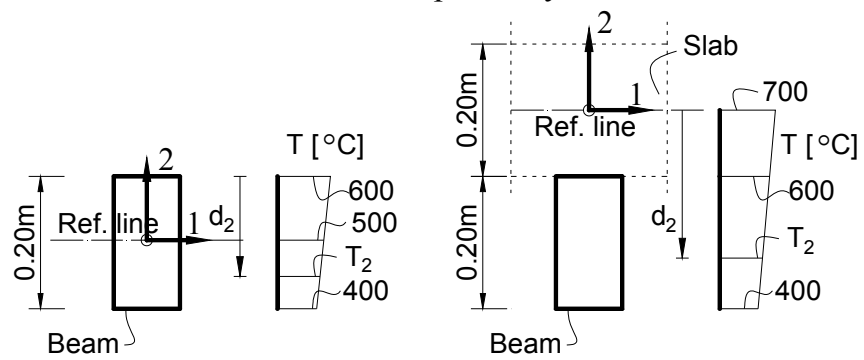

Figure 4: Reference line temperature for centroid position and arbitrary position.

For a simple example of a beam cross section as presented in Figure 4, it may be obtained the temperature increase of the beam cross section points. If the analysis is considered to be transient the temperature field is obtained with respect to time as presented in Figure 5. The two reference line position should lead to the same values of section point temperature if defined as mentioned before for each case with different values for temperature gradient.

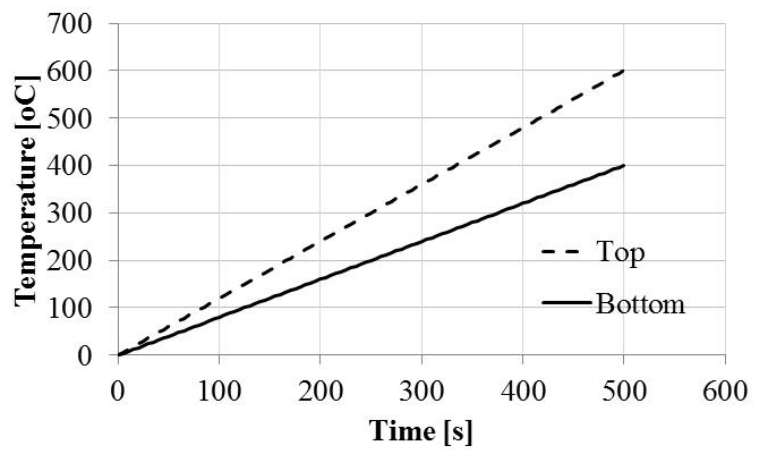

Figure 5: Temperature field in beam section points.

It must also be mentioned that for analysis which involves thermal gradient definition Abaqus considers the TEMP variables while for other temperature load, constant or input data, the nodal temperatures, NT, are used. The relevance is given for cases when the user tries to match the temperatures for different cases of thermal load definition as gradient, constant or input data. 


\subsection{Concrete model}

The use of concrete material models available in Abaqus may be found in Chaudhari and Chakrabarti [11]. The constitutive law of concrete damaged plasticity is generically presented for tensile stress in Figure 6(a) and for compressive behaviour in Figure 6(b). This concrete model has the advantages of decreasing branches for both tension and compression stresses and may be defined as temperature dependent.

Fire design Eurocode for concrete material [9] provides the reduction coefficients needed to be used in concrete elements design. These values are presented for tension and compression in Table 1.

Table 1: Reduction factors for concrete strength.

\begin{tabular}{|r|c|c|c|c|c|c|c|c|c|c|c|}
\hline$\theta\left[{ }^{\circ} \mathrm{C}\right]$ & 20 & 100 & 200 & 300 & 400 & 500 & 600 & 700 & 800 & 900 & 1000 \\
\hline Compr. & 1.00 & 1.00 & 0.95 & 0.85 & 0.75 & 0.6 & 0.45 & 0.3 & 0.15 & 0.08 & 0.04 \\
\hline Tens. & 1.00 & 1.00 & 0.80 & 0.60 & 0.40 & 0.20 & 0.0 & 0.0 & 0.0 & 0.0 & 0.0 \\
\hline
\end{tabular}

Although design codes do not impose the use of tensile strength of concrete, for more accurate results and behaviour study of elements subjected to elevated temperatures, tensile resistance of concrete is considered. Also, when using concrete damaged plasticity model in Abaqus, numerical problems may occur if the tensile strain of concrete has a small value. Defining strain for the tensile behaviour of concrete comparable to the reinforcement strain is shown to be of neglectable importance [3].
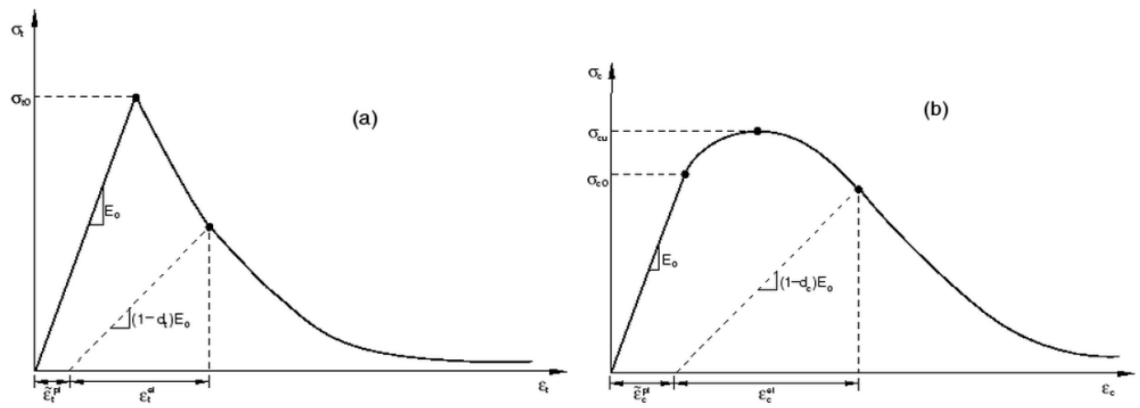

Figure 6: Response of concrete to uniaxial loading in (a) tension, (b) compression [3].

The general purpose computer code Abaqus has a default setting regarding the decreasing branches for tensile and compression branches. These intervals, tension stiffening and compression hardening respectively, are limited by a value of 0.01 of the maximum values on each branch. These values are reasonable and of less importance if the analysis is performed for ambient temperature. Although not specifically mentioned in the software documentation, the 
constraint of minimum $1 \%$ is also related to the decreasing of mechanical properties due to temperature.

It can be noticed in Table 1 that compressive strength at high temperature is not decreasing below $1 \%$ of the ambient compressive strength. The discussion is made on the tensile strength of concrete for high temperatures. Starting from $600^{\circ} \mathrm{C}$ the concrete is considered to completely lose the tensile strength. Since Abaqus is set by default to consider a minimum value for the tensile strength, it is important to assess its influence.

The area of importance for the concrete model considerations is to be established. Certainly, the compressed elements will show no difference if the tensile parameters are modified. The effect should be visible in elements subjected to bending or tension. Although not very common for concrete elements, tensioned elements may appear in composite slabs subjected to elevated temperatures due to the tensile membrane action (TMA).

A simple model using shell elements for a $2 \mathrm{~m}$ long concrete slab strip $(1.0 \mathrm{x}$ $0.2) \mathrm{m}^{2}$ subjected to bending is defined with two different values for tensile strength for ambient temperature $\left(3 \mathrm{~N} / \mathrm{mm}^{2}\right.$ and $\left.0.1 \mathrm{~N} / \mathrm{mm}^{2}\right)$. The mechanical load is defined in a first step by a pressure of $40000 \mathrm{~N} / \mathrm{m}^{2}$. The thermal load is defined as constant temperature in a second step of $1000 \mathrm{~s}$ with a linear increase from $0^{\circ} \mathrm{C}$ to $1000^{\circ} \mathrm{C}$. The choice of constant temperature definition is taken in order to avoid uncertainties of temperature field in case of gradient thermal field with respect to time.

Usually an anti-cracking reinforcement layer is considered in the middle plane of cross-section. The yield stress of steel is linearly decreasing from $500 \mathrm{~N} / \mathrm{mm}^{2}$ at $0^{\circ} \mathrm{C}$ to $0 \mathrm{~N} / \mathrm{mm}^{2}$ at $1000^{\circ} \mathrm{C}$.

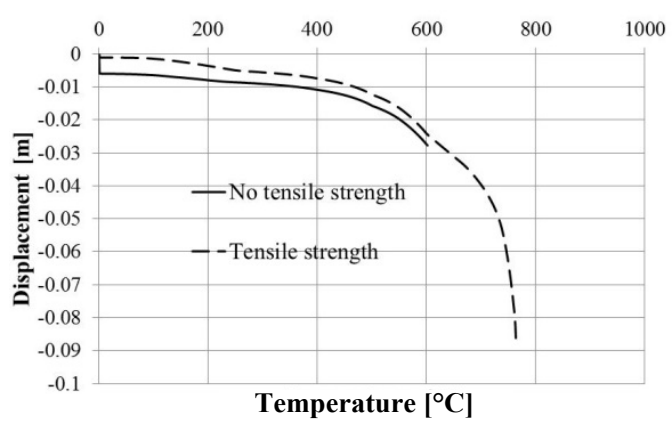

(a)

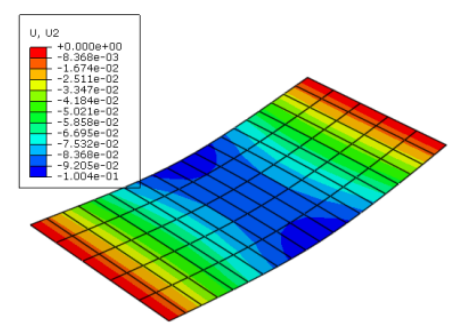

(b)

Figure 7: (a) Midspan displacement of slab; (b) deformed shape of slab.

The displacements resulted from analysis of the two cases are depicted graphically in Figure 7(a). It may be observed that the difference between enhancing tensile stress and excluding it is in both displacement and temperature domain. Since temperature is developed in time, it may also influence the fire resistance of an element in the time domain. 
Difference in slab deflection disregarding tensile strength is greater than in the case of defining it. The difference tends to be smaller as temperature increases. At the moment concrete reaches $600^{\circ} \mathrm{C}$, thus no tensile stress should develop, the value obtained with no tensile strength can be considered to be referenced. It may be observed that difference between the two cases is less than $1 \%$.

In the temperature/time domain the chart exhibits an extension of fire resistance above $600^{\circ} \mathrm{C}$. This part may be considered non-conservative for composite slabs which develop the TMA stage. Although in real composite slabs, temperatures reach $600^{\circ} \mathrm{C}$ only in a small thickness from the total height of the slab special attention should be paid to the influence over the shallow concrete slabs.

The example presented is constructed only to point out the influence of tensile strength of concrete at elevated temperature. Due to the large number of thermal loads in practice, elements structural configuration, thermal and mechanical material properties at high temperatures specific values for disparities cannot be established. Including the tensile strength and assessing its effect should be determined by a conservative and engineering judgement.

\section{Conclusions}

Analysis of structures at elevated temperature is highly dependent on the thermally induced effects by expansion coefficient. Either constant temperature or thermal gradient are considered by computer codes in different manners.

The paper presents the approaches of Abaqus with respect to the theoretical relations in the literature. Analysis which involves temperature dependent materials must be performed considering the initial temperature for thermal expansion coefficient. In situations of different temperature over the crosssection, definition of thermal gradient must consider the position of reference line of the element.

Concrete damaged plasticity model in Abaqus has a default setting constraining the minimum stress of decreasing tensile/compressive branches which is limited not only for the same temperature behaviour but also for maximum stresses at different temperatures. Function of the parameters involved in analysis the minimum setting of stress in Abaqus might influence the response of an element in both displacement and temperature domain.

\section{Acknowledgements}

This paper was supported by the European social fund within the framework of realizing the project Support of inter-sectoral mobility and quality enhancement of research teams at Czech Technical University in Prague, CZ.1.07/2.3.00/30.0034.

This paper was also supported by grant of Grant Agency Czech Republic, Models of steel and fibre concrete composite columns exposed to fire, GACR 15-19073S. 


\section{References}

[1] Franssen, J-M., Safir-A thermal/structural program modelling structures under fire, Engineering Journal, pp. 143-158, 2005.

[2] Vulcan, http://www.vulcan-solutions.com/

[3] Abaqus, Abaqus Documentation 6.11. Providence, RI, USA: Dassault Systèmes, 2011.

[4] ANSYS, Ansys Inc., 2008.

[5] Zaharia, R., Vulcu, C., Vassart, O., Gernay, T., \& Franssen, J-M., Numerical analysis of partially fire protected composite slabs, Steel and Composite Structures, pp. 21-39, 2013.

[6] Huang, Z., Burgess, I., \& Plank, R.J., Modeling Membrane Action of Concrete Slabs in Composite Buildings in Fire. II: Validations, Journal of Structural Engineering, vol. 129, no. 8, pp. 1103-1112, 2003.

[7] Zhao, B., \& Roosefid, M., Experimental and numerical investigations of steel and concrete composite floors subjected to ISO fire Condition, Proceedings of the Sixth International Conference Structures in Fire, eds. V. Kodur, J-M. Franssen, DEStech Publications, Inc.: East Lansing, pp. 407-416, 2010.

[8] EN 1994-1-2, European Committee for Standardization, Eurocode 4 Design of composite steel and concrete structures - Part 1-2: General rules - Structural fire design, 2004.

[9] EN 1992-1-2, European Committee for Standardization, Eurocode 2 Design of concrete structures - Part 1-2: General rules - Structural fire design, 2005.

[10] EN 1993-1-2, European Committee for Standardization, Eurocode 2 Design of steel structures - Part 1-2: General rules - Structural fire design, 2005.

[11] Chaudhari. S.V. \& Chakrabarti, M.A., Modeling of concrete for nonlinear analysis Using Finite Element Code ABAQUS, International Journal of Computer Applications, vol. 44, no. 7, pp. 14-18, 2012. 